Revue de droit comparé du travail et de la sécurité sociale

1 | 2020

Doctrine

\title{
Livret de travail : un nouveau regard sur un document familier
}

\section{Elena Serebryakova}

\section{(2) OpenEdition}

1 Journals

Édition électronique

URL : https://journals.openedition.org/rdctss/1259

DOI : $10.4000 /$ rdctss. 1259

ISSN : 2262-9815

Éditeur

Centre de droit comparé du travail et de la sécurité sociale

\section{Édition imprimée}

Date de publication : 1 avril 2020

Pagination : 180-183

ISSN : 2117-4350

\section{Référence électronique}

Elena Serebryakova, "Livret de travail : un nouveau regard sur un document familier », Revue de droit comparé du travail et de la sécurité sociale [En ligne], 1 | 2020, mis en ligne le 01 novembre 2021, consulté le 11 novembre 2021. URL : http://journals.openedition.org/rdctss/1259 ; DOI : https:// doi.org/10.4000/rdctss. 1259

\section{(c) (i) $\odot$}

Revue de droit comparé du travail et de la sécurité sociale est mise à disposition selon les termes de la Licence Creative Commons Attribution - Pas d'Utilisation Commerciale - Pas de Modification 4.0 International. 


\section{ELENA SEREBRYAKOVA}

UNIVERSITÉ NATIONALE DE RECHERCHE «ÉCOLE DES HAUTES ÉTUDES

EN SCIENCES ÉCONOMIQUES »

\section{LIVRET DE TRAVAIL : UN NOUVEAU REGARD SUR UN DOCUMENT FAMILIER}

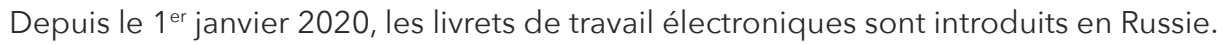

L'histoire des livrets de travail en Russie remonte à des décennies. Introduits pour la première fois en octobre 1918 et appelés « certificat de travail temporaire pour la bourgeoisie ", ces documents étaient destinés aux personnes "non actives ", comme par exemple les chefs de sociétés par actions, les rentiers, les commerçants privés. Le certificat temporaire consistait en une carte d'identité dans laquelle il était noté chaque mois l'exécution du travail d'intérêt général effectué par son propriétaire. Sans ces précisions, le document était invalide et il était impossible de se procurer des denrées alimentaires. En juin 1919, les livrets de travail sont devenus obligatoires à Moscou et à Petrograd (devenue Saint-Pétersbourg) pour tout citoyen, dès l'âge de 16 ans, quel que soit leur emploi. Le livret de travail était ainsi délivré comme passeport et servait de carte d'identité, donnant non seulement le droit de se déplacer mais aussi d'obtenir de la nourriture et d'être inscrit à la sécurité sociale. En 1923, à l'ère de la nouvelle politique économique, les livrets de travail furent alors remplacés par les cartes d'identité.

L'introduction de livrets de travail en URSS s'est faite par le biais d'un décret gouvernemental de décembre 1938. Il est devenu un document obligatoire pour les travailleurs ainsi que pour les salariés des entreprises d'État et coopératives. Ce document unique ne prouvait pas l'identité, mais contenait différentes informations sur le travail. Le livret de travail est alors devenu un document obligatoire pour l'emploi. Une exception à cette règle a été établie pour les personnes lors de leur première embauche. Dans ce cas, ce livret était délivré par le premier employeur. Le livret de travail du salarié était conservé par l'employeur. Il y était inscrit des informations sur le travail en tant que tel (changements d'emploi, licenciement du salarié, motifs de rupture du contrat de travail...). Le livret de travail faisait ainsi office de curriculum vitae et contenait toutes les indications nécessaires à l'attribution des prestations de sécurité sociale et de retraite.

L'ensemble de ces règles générales s'appliquent encore aujourd'hui. Selon l'article 66 du Code du travail de la Fédération de Russie, le livret de travail reste en Russie le principal document sur l'activité et l'expérience de travail d'un salarié. Lors de son embauche, chaque salarié doit obligatoirement présenter à l'employeur son livret de travail - sauf en cas de première embauche ou d'embauche pour un emploi secondaire. Dans ce dernier cas, le livret de travail est conservé sur le lieu principal de travail. Enfin, un livret de travail perdu doit être refait.

La tenue de livrets de travail n'est pas une tâche facile pour l'employeur, qui doit disposer d'une réserve de formulaires vierges de livrets de travail conservés dans un coffre-fort. Les notations consignées dans le livret de travail sont effectuées conformément aux règles édictées par le Ministère du travail et du développement social, et leur modification n'est possible qu'à condition d'annuler une mauvaise note pour en décerner 
une nouvelle. L'employeur doit informer le salarié de toute note inscrite dans son livret de travail. Notons également que les livrets de travail non réclamés, y compris ceux des travailleurs décédés, sont conservés par l'employeur pendant 75 ans.

Depuis le $1^{\text {er }}$ janvier 2020, la collecte de données électroniques sur l'activité professionnelle des travailleurs a été établie en Russie avec l'entrée en vigueur des lois $\mathrm{n}^{\circ} 439-\mathrm{FZ}^{1}$ et $436-\mathrm{FZ}^{2}$ du 16 décembre 2019. Les informations relatives à l'activité professionnelle comprennent des indications personnalisées sur le lieu de travail, la fonction de travail, les changements d'emploi, les motifs de la rupture du contrat de travail.

Au cours de cette année 2020, les salariés ${ }^{3}$ auront le droit de choisir l'une des deux façons de recenser ces informations sur leur activité professionnelle : via un livret de travail sur papier traditionnel ou par voie électronique. Les personnes souhaitant conserver ces informations uniquement sous forme électronique recevront leurs livrets de travail sur papier des employeurs. À l'avenir, ils pourront obtenir de manière indépendante différentes informations concernant leur travail, en particulier grâce au service électronique de la Caisse des pensions, en contactant le Centre plurifonctionnel des services publics et municipaux, ou encore l'employeur du dernier lieu de travail. Néanmoins, l'employeur ne fournit des informations que pour la période de travail exercée dans son entreprise. Le fait qu'un employeur continue à tenir des livrets de travail « papier» ne le dispense pas de son obligation de transmettre à la Caisse des pensions les informations sur l'activité de travail de tous ses salariés. Par conséquent, si le salarié ne rédige pas de déclaration donnant son consentement pour une gestion des informations sous forme électronique, l'employeur devra non seulement tenir le livret de travail de ce salarié, mais également communiquer les informations à la Caisse des pensions.

Durant cette année 2020, les employeurs vont être amenés à prendre différentes mesures permettant d'assurer la transmission des informations professionnelles de chaque salarié à la Caisse des pensions: embauche, changement d'emploi, licenciement, et choix du salarié pour le livret de travail " papier » ou sous forme électronique, etc. Les informations notées doivent être transmises par l'employeur au plus tard le quinzième jour du mois suivant celui au cours duquel les changements professionnels décrits ci-avant se sont produits; mais, en l'absence de telles modifications, l'employeur a jusqu'au 15 février 2021 pour transmettre à la Caisse des pensions les informations nécessaires.

Les employeurs qui emploient moins de 25 salariés ont la possibilité d'envoyer des informations sur papier à la Caisse des pensions. Les autres entreprises doivent fournir ces informations sous forme électronique avec une signature électronique certifiée. En cas de non-présentation des informations concernant les travailleurs, d'informations fausses ou incomplètes, l'employeur peut être tenu administrativement responsable d'une violation de la législation du travail, passible d'une amende de 30000 à 50000 roubles (de 430 à

1 Loi fédérale du 16 décembre 2019 n439-Ф3 portant modification du Code du travail de la Fédération de Russie en ce qui concerne la production d'informations sur les activités de travail sous forme électronique : http: //pravo.gov.ru/proxy/ips/?docbody=\&nd=102640677\&intelsear ch $=439-\%$ D $4 \%$ C7

2 Loi fédérale du 16 décembre 2019 n436-\$3 portant modification de la loi fédérale sur la " comptabilité individuelle (personnifiée) dans le système d'assurance pension obligatoire »: http://pravo.gov.ru/proxy/ips/ ?docbody =\&nd=102640671\&intelsearch=436-\%D4\%C7

3 Y compris les fonctionnaires de l'État et les agents municipaux. 
715 euros environ). De plus, si le motif du licenciement signalé à la Caisse des pensions est non-conforme au Code du travail, l'employeur devra indemniser le salarié à hauteur d'un montant égal au salaire perdu.

Néanmoins, les dispositions modifiées du Code du travail ne sont pas sans défauts. L'article 65 du Code du travail de la Fédération de Russie prévoit que, lors de l'établissement d'un contrat de travail, le salarié présente à l'employeur, entre autres documents, un livret de travail et/ou des informations sur son activité professionnelle. Sur la base de l'interprétation littérale de cette règle, l'employeur a le droit d'exiger du salarié la présentation de ces documents. Or, conformément aux articles 66.1 et 84.1 du Code du travail de la Fédération de Russie, les informations professionnelles ne sont délivrées par l'employeur qu'au salarié dont le livret de travail n'a pas été tenu. Par conséquent, un salarié dont le livret de travail était tenu peut ne pas disposer des informations professionnelles au moment de son embauche chez un nouvel employeur. Pour les obtenir, il devra contacter le centre des prestations des services publics ou la Caisse des pensions.

De plus, les difficultés liées à l'introduction de changements dans les informations portant sur l'activité professionnelle ne sont pas complètement résolues. Conformément à l'article 66.1 du Code du travail de la Fédération de Russie, les informations incorrectes sur l'activité professionnelle sont corrigées par l'employeur, sur demande écrite du salarié. Pour modifier les informations, l'employeur doit envoyer un rapport correspondant à la Caisse des pensions. Cependant, une telle modification peut être effectuée non seulement sur la base de la demande du salarié, mais également sur décision de justice, par exemple lorsque le salarié réfute le motif de son licenciement et le conteste devant le tribunal. Dans ce cas, l'enregistrement de la modification du motif de licenciement dans le livret de travail sur papier est effectué par l'employeur - puisque c'est lui qui tient ce livret de travail - mais il n'est pas le détenteur des données électroniques sur les activités de travail, ces dernières étant collectées par la Caisse des pensions. Une décision de justice ayant autorité de la chose jugée pour modifier le libellé d'un motif de licenciement est donc obligatoire à tous les niveaux (employeur, Caisse des pensions, centre des prestations des services publics). Cependant, à ce jour, la loi ne prévoit pas la possibilité de modifier les informations sur l'activité professionnelle par une décision de justice sans la participation de l'employeur.

À ce jour, il est difficile de prédire le nombre de travailleurs qui vont refuser de continuer à utiliser le livret de travail sur papier. Les employeurs favorisent davantage la transition des salariés vers la collecte électronique des informations professionnelles. En pratique, les salariés n'ont aucun intérêt à abandonner les livrets de travail. Pour beaucoup d'entre eux, il est plus facile et plus compréhensible d'utiliser des documents familiers qui reflètent toutes leurs informations professionnelles depuis le début de leur carrière, tandis que les informations électroniques ne sont générées que depuis le 1 er janvier 2020. En outre, l'article 66 du Code du travail de la Fédération de Russie prévoit que le livret de travail sur papier est le document principal concernant l'activité et l'expérience de travail du salarié. A l'inverse, parmi les avantages de la comptabilité électronique des informations sur les activités du travail, appelées le « livret de travail électronique», la Caisse des pensions met en avant ${ }^{4}$ : un accès pratique et rapide des travailleurs à ces informations; des opportunités supplémentaires pour l'emploi à distance; la réduction des coûts supportés par les employeurs pour l'acquisition, la tenue et le stockage des livrets de travail sur papier ; la

4 Voir l'information sur site de la Caisse des pensions : http://www.pfrf.ru/etk\#info-1 


\section{FÉdérATION DE RUSSIE}

possibilité de constituer à distance les retraites selon le compte personnel de chacun sans apporter la preuve de documentaires supplémentaires.

Toutefois, les raisons invoquées par l'Etat pour collecter toutes les données sur le travail de chaque personne ne sont pas toujours très convaincantes. Ainsi, le motif de la rupture du contrat de travail n'a pas d'importance pour l'enregistrement des pensions. Force est de constater que, depuis plus de 80 ans d'existence, les livrets de travail sur papier se sont imposés comme une source fiable d'informations sur le travail. Dès lors, les motivations liées à l'introduction d'un registre électronique centralisé de ces informations ne semblent pas suffisamment étayées. 